\title{
Combinatorial Methylerythritol Phosphate Pathway Engineering and Process Optimization for Increased Menaquinone-7 Synthesis in Bacillus subtilis
}

\author{
Taichi Chen ${ }^{1,2 \dagger}$, Hongzhi Xia ${ }^{3 \dagger}$, Shixiu Cui ${ }^{1}$, Xueqin Lv ${ }^{1}$, Xueliang Li ${ }^{1}$, Yanfeng Liu ${ }^{1}$, Jianghua Li ${ }^{2}$, \\ Guocheng $\mathrm{Du}^{1}$, and Long Liu ${ }^{1 *}$ \\ 'Key Laboratory of Carbohydrate Chemistry and Biotechnology, Ministry of Education, Jiangnan University, Wuxi \\ 214122, P.R. China \\ ${ }^{2}$ Key Laboratory of Industrial Biotechnology, Ministry of Education, Jiangnan University, Wuxi 214122, P.R. China \\ ${ }^{3}$ Richen Bioengineering Co., Ltd., Nantong 226000, P.R. China
}

\begin{abstract}
Vitamin K2 (menaquinone) is an essential vitamin existing in the daily diet, and menaquinone-7 (MK7) is an important form of it. In a recent work, we engineered the synthesis modules of MK-7 in Bacillus subtilis, and the strain BS20 could produce $360 \mathrm{mg} / \mathrm{l} \mathrm{MK-7} \mathrm{in} \mathrm{shake} \mathrm{flasks,} \mathrm{while} \mathrm{the}$ methylerythritol phosphate (MEP) pathway, which provides the precursor isopentenyl diphosphate for MK-7 synthesis, was not engineered. In this study, we overexpressed five genes of the MEP pathway in BS20 and finally obtained a strain (BS20DFHG) with MK-7 titer of $415 \mathrm{mg} / \mathrm{l}$ in shake flasks. Next, we optimized the fermentation process parameters (initial pH, temperature and aeration) in an 8-unit parallel bioreactor system consisting of $300-\mathrm{ml}$ glass vessels. Based on this, we scaled up the MK-7 production by the strain BS20DFHG in a 50-I bioreactor, and the highest MK-7 titer reached 242 $\mathrm{mg} / \mathrm{l}$. Here, we show that the engineered strain BS20DFHG may be used for the industrial production of MK-7 in the future.
\end{abstract}

Keywords: Menaquinone, methylerythritol phosphate pathway, Bacillus subtilis, process optimization

Received: December 5, 2019 Accepted: February 17, 2020

First published online: February 18,2020

*Corresponding author Phone: +86-510-85918312 Fax: +86-510-85918309 E-mail: longliu@jiangnan.edu.cn

These authors contributed equally.

Supplementary data for this paper are available on-line only at http://jmb.or.kr.

pISSN 1017-7825 eISSN 1738-8872

Copyright(C) 2020 by The Korean Society for Microbiology and Biotechnology

\section{Introduction}

Vitamin K2 (menaquinone, MK) is a series of compounds that share the 2-methyl-1,4-naphthoquinone but with different lengths and saturation degrees of the polyisoprene side chain attached to the 3-position. Among these compounds is menaquinone-7 (MK-7), which is the term for 2-methyl-3-heptaprenyl-1,4-naphthoquinone, meaning the polyisoprene side chain consists of seven prenyl units [1]. MK-7 can be directly absorbed and utilized by the human body and has a longer half-life in human blood than other forms of vitamin K [2]. Addition of MK7 in the daily diet can prevent bone fractures [2], treat vitamin K deficiency hemorrhagic disease [3], or prevent arterial calcification [4]. The biologically active MK-7 is mainly produced by microbial fermentation, including solid-state fermentation and liquid fermentation $[5,6]$. The solid-state fermentation takes natto (fermented soybean) as the substrate, and the reported maximum yield is $67 \mathrm{mg} / \mathrm{kg}$ by Bacillus subtilis natto [7]. The parameters of solid-state fermentation are challenging to control, and a large amount of metabolic heat is generated, resulting in the slow growth of bacteria and low MK-7 production. Therefore, increasing attention is being paid to the production of MK-7 by liquid fermentation, and the strains used in this method include Bacillus subtilis natto, Bacillus subtilis licheniformis, Flavobacterium, etc. [8-10]. Previous studies have explored the effects of biofilm growth, dissolved oxygen changes, $\mathrm{pH}$ and electron generation on the production of MK-7 [2, 11-13]. In recent work, we engineered the synthesis pathway of MK-7 in B. subtilis using a quorum-sensing system, and the highest titer of MK-7 reached $360 \mathrm{mg} / \mathrm{l}$ in shake flasks and $200 \mathrm{mg} / \mathrm{l}$ in the 15-1 bioreactor [14]

B. subtilis is Generally Recognized as Safe (GRAS) and is a typical gram-positive bacterium. The biosynthesis of MK-7 precursors in B. subtilis can be divided into two modules (Fig. 1). Naphthoquinone, derived from chorismic acid, is the bone structure of MK-7. The polyisoprene side chain is synthesized from glyceraldehyde-3-phosphate (G3P) and pyruvate (PYR) through the MEP pathway. Then, the polyisoprene side chain will be joined to the naphthoquinone to form demethylmenaquinone (DMK) by 1, 4-dihydroxy-2-naphthoate octaprenyltransferase (MenA). Finally, MK-7 is synthesized when the methylation of DMK is finished. In other recent work, we developed a bifunctional quorum-sensing system in B. subtilis 168 to engineer the synthesis modules of MK-7, and obtained a recombinant strain BS20 [14], while the MEP pathway had not been engineered. There are many studies about the MEP pathway and its final product, isopentenyl diphosphate (IPP). The accumulation of IPP can 


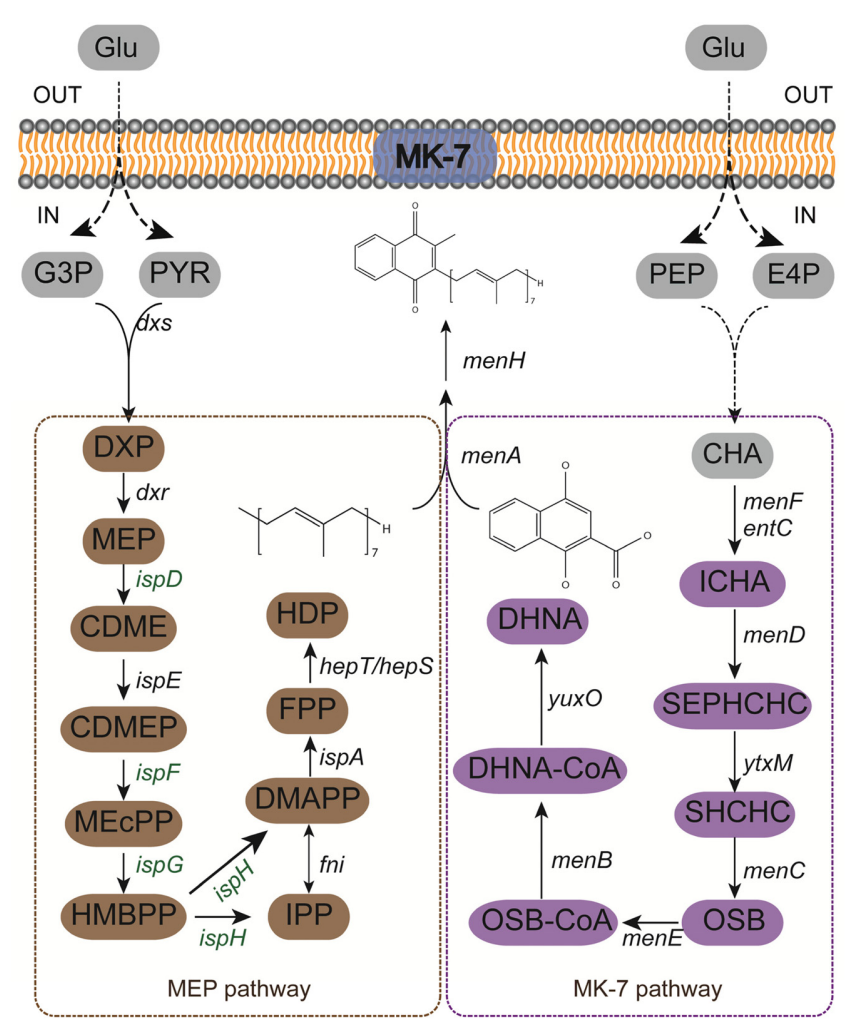

Fig. 1. The pathway of MK-7 biosynthesis. MK-7 is synthesized from two precursors that come from MEP pathway and MK-7 pathway, respectively. The dashed lines mean multiple reactions and the genes in green are the overexpressed genes in the strain BS20DFHG. Enzymes: Dxs, 1-deoxyxylulose-5-phosphate synthase; Dxr, 1-deoxyxylulose-5-phosphate reductoisomerase; IspD, 2-C-methylerythritol 4-phosphate cytidylyltransferase; IspE, 4-diphosphocytidyl-2-C-methylerythritol kinase; IspF, 2C-methylerythritol 2,4-cyclodiphosphate synthase; IspG, 4-hydroxy-3-methylbut-2-enyl diphosphate synthase; IspH, 4hydroxy-3-methylbut-2-enyl diphosphate reductase; Fni, isopentenyl diphosphate isomerase; IspA, farnesyl diphosphate synthase; HepS/HepT, heptaprenyl diphosphate synthase component I/II. MenF, isochorismate synthase; EntC, isochorismate synthase from E. coli K12; MenD, 2-succinyl-5-enolpyruvyl-6-hydroxy-3-cyclohexene-1-carboxylate synthase; YtxM, 2succinyl-6-hydroxy-2,4-cyclohexadiene-1-carboxylate synthase; MenC, o-succinylbenzoate synthase; MenE, o-succinylbenzoic acid-CoA ligase; MenB, 1,4-dihydroxy-2-naphthoyl-CoA synthase; YuxO, 1,4-dihydroxy-2-naphthoyl-CoA hydrolase; MenA, 1,4-dihydroxy-2-naphthoate heptaprenyltransferase; $\mathrm{MenH}$, demethylmenaquinone methyltransferase. Abbreviation of metabolites: Glu, glucose; PEP, phosphoenolpyruvate; PYR, pyruvate; E4P, erythrose 4-phosphate; G3P, glyceraldehyde-3phosphate; CHA, Chorismite; ICHA, isochorismate; SEPHCHC, 2-succinyl-5-enolpyruvyl-6-hydroxy-3-cyclohexene-1carboxylate; SHCHC, 2-succinyl-6-hydroxy-2,4-cyclohexadiene-1-carboxylate; OSB, 2-succinylbenzoate; OSB-CoA, 2succinyl benzoyl-CoA; DHNA-CoA, 1,4-dihydroxy-2-naphthoyl-CoA; DHNA, 1,4-dihydroxy-2-naphthoate; DXP, 1deoxyxylulose-5-phosphate; MEP, methyl-erythritol-4-diphosphate; CDME, methylerythritol cytidyl diphosphate; CDMEP, 4-diphosphocytidyl-2-C-methyl-D-erythritol-2-phosphate; MEcPP, 2-C-methyl-D-erythritol-2,4-cyclodiphosphate; HMBPP, 1-hydroxy-2-methyl-2-butenyl 4-diphosphate; DMAPP, dimethylallyl diphosphate; IPP, isopentenyl diphosphate; FPP, farnesyl diphosphate; HDP, heptaprenyl diphosphate; MK-7, menaquinone-7.

increase the production of many natural products such as menaquinone, ubiquinone, carotenoids and taxadiene [15-19]. 1-deoxyxylulose-5-phosphate synthase (Dxs) and 1-deoxyxylulose-5-phosphate reductoisomerase (Dxr) are thought to catalyze rate-limiting steps of the MEP pathway. Up-regulating Dxs and Dxr in B. subtilis can significantly improve the production of MK-7 [5]. The titer of carotenoids in B. subtilis was increased by engineering the five enzymes (IspD - IspH) catalyzing MEP to IPP and dimethylallyl diphosphate (DMAPP) [16].

In this study, we focused on enhancing the transcription level of five isp-genes (ispD, ispE, ispF, ispH, and ispG) in the MEP pathway to promote the production of MK-7. The highest MK-7 production of $415 \pm 3.2 \mathrm{mg} / \mathrm{l}$ was observed in shake culture of the engineered strain BS20DFHG. Then, we conducted fermentation optimization in an 8-unit, 300-ml bioreactor system, and the highest titer of MK-7 was $185.57 \pm 6.3 \mathrm{mg} / \mathrm{l}$ at $41^{\circ} \mathrm{C}, 2 \mathrm{vvm}$ and an initial $\mathrm{pH}$ of 7.0. Finally, we conducted fermentation in a $50-1$ bioreactor under optimized conditions, and $242 \pm$ $5.5 \mathrm{mg} / \mathrm{l}$ of MK-7 was produced.

\section{Materials and Methods}

\section{Microorganisms and Reagents}

All the constructed B. subtilis strains and plasmids in this study are listed in Table 1 . All microorganisms were cultivated in Luria-Bertani (LB) liquid culture or on LB agar plates at $37^{\circ} \mathrm{C}$ for genetic experiments. The 
Table 1. Strains and plasmids used in this study.

\begin{tabular}{|c|c|c|}
\hline Names & Characteristics & Reference \\
\hline \multicolumn{3}{|l|}{ Strains } \\
\hline BS20 & 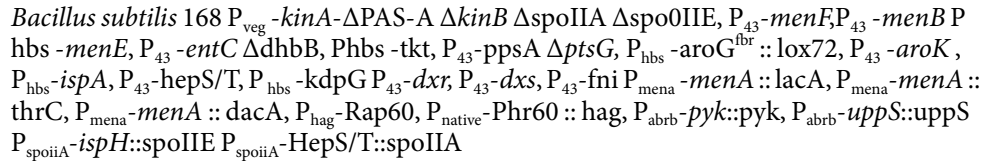 & Lab stock \\
\hline BS20D & $\mathrm{BS} 20 \mathrm{P}_{43}-i s p D:: i s p D$ & This study \\
\hline BS20DF & $\mathrm{BS} 20 \mathrm{D} \mathrm{P}_{43}-i s p F:: i s p F$ & This study \\
\hline BS20DFE & $\mathrm{BS} 20 \mathrm{DF} \mathrm{P}_{43}-i s p E::$ ispE & This study \\
\hline BS20DFH & $\mathrm{BS}_{20 \mathrm{DF}} \mathrm{P}_{43}-i s p H:: i s p H$ & This study \\
\hline BS20DFHG & BS20DFH $\mathrm{P}_{43}-i s p G:: i s p G$ & This study \\
\hline \multicolumn{3}{|l|}{ Plasmids } \\
\hline P7C6 & Pmd18-T containing lox71-zeo-lox66 cassette & Lab stock \\
\hline PDG148 & Amp, Km, E. coli-B. subtilis shuttle vector, containing cre under the control of $\mathrm{P}_{\text {spac }}$ & Lab stock \\
\hline
\end{tabular}

fermentation medium for shake flasks consisted of $5 \%(\mathrm{w} / \mathrm{v})$ glucose, $5 \%(\mathrm{w} / \mathrm{v})$ soy peptone, $5 \%(\mathrm{w} / \mathrm{v})$ sucrose and $0.06 \%(\mathrm{w} / \mathrm{v}) \mathrm{KH}_{2} \mathrm{PO}_{4}$. Culture media were sterilized for $20 \mathrm{~min}$ at $115^{\circ} \mathrm{C}$. Reagents mentioned above were purchased from Sinopharm Chemical Reagent Co., Ltd. (Shanghai, China). Appropriate antibiotics were added into the medium: kanamycin $(50 \mu \mathrm{g} / \mathrm{ml})$, zeocin $(20 \mu \mathrm{g} / \mathrm{ml})$, ampicillin $(100 \mu \mathrm{g} / \mathrm{ml})$, spectinomycin $(50 \mu \mathrm{g} / \mathrm{ml})$, and chloramphenicol $(5 \mu \mathrm{g} / \mathrm{ml})$. All antibiotics were purchased from Sangon Biotech Co., Ltd. (China). A standard sample of MK-7 was purchased from ChromaDex (USA). Methanol, dichloromethane, 2-propanol, and n-hexane were obtained from Sigma-Aldrich (USA).

\section{In Situ Replacement of Promoter}

The expression of isp-genes was strengthened by replacing the native promoter with the strong constitutive promoter $\mathrm{P}_{43}$. The homogenous arms of isp-genes, target sequences and $\mathrm{P}_{43}$ promoter sequences were amplified from $B$. subtilis. The modified DNA fragments and plasmids were sequenced by GENEWIZ (USA). Primers used for genetic engineering were listed in Table S1. These three fragments and the lox71-resistance marker-lox66 cassettes were combined by overlapping PCR. The purified PCR products were used to transform competent B. subtilis cells by electroporation. The PDG148 plasmid was then transformed into antibiotic-resistant clones to promote the recombination of lox71 and lox66, which evicted the resistance marker cassette as previously described [20]. Finally, the intracellular plasmid PDG148 was lost by incubation at $50^{\circ} \mathrm{C}$ for $12 \mathrm{~h}$.

\section{Shake-Flask Culture of the Engineered B. subtilis}

A ring of engineered $B$. subtilis strains was picked from the plate and inoculated into $3 \mathrm{ml} \mathrm{LB}$ at $37^{\circ} \mathrm{C}$ with shaking at $220 \mathrm{rpm}$ for $6 \mathrm{~h}$ in a 15-ml tube. Each 250-ml Erlenmeyer flask contained $20 \mathrm{ml}$ of fermentation medium, which was inoculated with $1 \mathrm{ml}$ of seed liquid and grown at $41^{\circ} \mathrm{C}$ with shaking at $220 \mathrm{rpm}$ for 6 days. In addition, $500 \mu \mathrm{l}$ of fermentation broth was taken every $24 \mathrm{~h}$ for MK-7 concentration analysis.

\section{MK-7 Extraction}

MK-7 was extracted from the fermentation broth by extracting agent, which was a mixture of 2-propanol and nhexane $(1: 2, \mathrm{v} / \mathrm{v})$ in a 4:1 ratio (organic: liquid, $\mathrm{v} / \mathrm{v})$. The mixture was vigorously shaken with a vortex mixer for $10 \mathrm{~min}$ and then centrifuged at $7,000 \times \mathrm{g}$ for $5 \mathrm{~min}$ to separate two phases. The organic phase recovering extracted MK-7 was then separated as a fermentation sample for HPLC analysis.

\section{Analytical Methods}

Cell density was determined from the optical density at $600 \mathrm{~nm}$ with a spectrophotometer after suitable dilution with deionized water. The glucose concentration in the media was measured by a glucose-glutamate analyzer (SBA-40C; Biology Institute of Shandong Academy of Sciences, China). High-performance liquid chromatography (Agilent 1260, USA) equipped with a photon diode array UV detector was used to measure the MK-7 concentration of fermentation samples with a C18-ODS column $(5 \mu \mathrm{m}, 250 \times 4.6 \mathrm{~mm}$, Thermo Fisher Scientific, USA) at $40^{\circ} \mathrm{C}$. The mobile phase consisting of methanol: dichloromethane $(9: 1, \mathrm{v} / \mathrm{v})$ was used at a flow rate of $1 \mathrm{ml} / \mathrm{min}$. A wavelength of $254 \mathrm{~nm}$ was used for calibration and analysis. The MK-7 calibration curve was linear between $1 \mathrm{mg} / \mathrm{l}$ and $100 \mathrm{mg} / \mathrm{l}\left(\mathrm{R}^{2}=0.998\right)$.

\section{Quantitative Reverse-Transcriptase PCR (qRT-PCR) Analysis}

Cells were harvested from shake-flask cultures and frozen immediately in liquid nitrogen for 10 min on the third day. Total RNA was purified by using the RNAprep Pure Kit (Tiangen Biotech, China) according to the manual. The quantity and purity of RNA were measured by absorbance measurements at 260 and $280 \mathrm{~nm}$ using a Nanodrop ND-1000 spectrophotometer (Thermo Scientific, USA). Subsequently, cDNA, which was obtained by reverse transcription of total RNA using a PrimeScript TM RT-PCR Kit (Takara, China), was used as the template for qPCRs. qPCR was performed in a 96-well plate with a total reaction volume of approximately $20 \mu \mathrm{l}$ using SYBR Premix Ex Taq TM (Takara) according to the manufacturer's specifications. The reactions were conducted with a 
LightCycler 480 II Real-time PCR instrument (Roche Applied Science, Mannheim, Germany). The hbs U gene was used as the internal standard [21].

\section{Fed-Batch Culture in 300-ml Bioreactors}

MK-7 fermentation by fed-batch culture of BS20DFHG was performed in 300-ml bioreactors (MiniBox, T\&J Bioengineering Co., Ltd. (Shanghai, China). The 'aeration' was selected as an influencing factor to simulate the industrial process. The fermentation media used for fed-batch culture consisted of $5 \%(\mathrm{w} / \mathrm{v})$ soy peptone, $0.06 \%$ $(\mathrm{w} / \mathrm{v}) \mathrm{KH}_{2} \mathrm{PO}_{4}$ and initial glucose concentration of $1.3 \%(\mathrm{w} / \mathrm{v})$ for each bioreactor. The feeding solution contained $500 \mathrm{~g} / \mathrm{l}$ glucose. Seed culture was cultured in $250-\mathrm{ml}$ shake flasks containing $50 \mathrm{ml}$ of seed medium with shaking at $220 \mathrm{rpm}$ and $37^{\circ} \mathrm{C}$ for $6 \mathrm{~h}$. The seed culture was inoculated into a $300-\mathrm{ml}$ bioreactor containing $170 \mathrm{ml}$ fermentation medium by sterile injectors. The initial $\mathrm{pH}$ was adjusted to 7.0 by ammonium hydroxide, and the temperature was maintained at $41{ }^{\circ} \mathrm{C}$. The $100 \%$ calibration is achieved by increasing agitation to approximate $600 \mathrm{rpm}$ and increasing airflow to $1 \mathrm{vvm}$. In fed-batch culture, when the residual glucose concentration fell below $0.7 \%(\mathrm{w} / \mathrm{v})$, the feeding solution was pumped into the fermenter to restore the glucose concentration to $0.6-1.5(\mathrm{w} / \mathrm{v})$. The feeding rates were adjusted every $2 \mathrm{~h}$ based on the concentration of residual glucose in the fermentation medium.

\section{Fed-Batch Culture in 50-1 Bioreactor}

MK-7 fermentation by fed-batch culture of BS20DFHG was performed in 50-1 bioreactors at Richen Bioengineering Co., Ltd., Nantong. It was also performed at an initial glucose concentration of $1.3 \%(\mathrm{w} / \mathrm{v})$. The fermentation medium used for the fed-batch culture consisted of $5 \%(\mathrm{w} / \mathrm{v})$ soy peptone and $0.06 \%(\mathrm{w} / \mathrm{v}) \mathrm{KH}_{2} \mathrm{PO}_{4}$. The feeding solution contained $50 \%(\mathrm{w} / \mathrm{v})$ glucose. Seed culture was carried out in 2-L shake flasks containing $700 \mathrm{ml}$ of seed medium with shaking at $220 \mathrm{rpm}$ and $37^{\circ} \mathrm{C}$ for $8 \mathrm{~h}$. The seed culture was inoculated into a $50-1$ fermenter containing $32 \mathrm{~L}$ fermentation medium. The $\mathrm{pH}$ was set at 7.0 in the beginning, and the temperature was maintained at $4^{\circ} \mathrm{C}$ in the whole process. The $100 \%$ calibration was achieved by increasing agitation to $450 \mathrm{rpm}$ and increasing airflow to $1 \mathrm{vvm}$. In fed-batch culture, when the residual glucose concentration fell below $1 \%(\mathrm{w} / \mathrm{v})$, the feeding solution was pumped into the fermenter to restore the glucose concentration to $1.2-1.5(\mathrm{w} / \mathrm{v})$. The feeding rates were adjusted every $2 \mathrm{~h}$ based on the concentration of residual glucose in the fermentation medium.

\section{Cell Growth Kinetic Model}

The kinetic parameters of fermentation equations (Eqs) were analyzed by Origin 2020 software. The differential equations were solved and plotted using MATLAB.

\section{Statistical Analysis}

All experiments were independently carried out at least three times, and the results were expressed as mean \pm standard deviation (SD). All the data shown were mean values and are based on the recorded data unless otherwise indicated.

\section{Results and Discussion}

\section{Increased MK-7 Production by MEP Pathway-Strengthened BS20DFHG Strain}

To increase the flux of the MEP pathway, we overexpressed isp-genes by replacing native promoters with $\mathrm{P}_{43}$ promoter stepwise. Then we conducted fermentation of the recombinant strains in 250-ml shake flasks for 6 days because the highest yield of MK-7 was reached on this day (Fig. S1). Moreover, the relative transcriptional level of isp-genes was detected on the third day of culture.

First, we replaced the promoter of the gene ispD with $\mathrm{P}_{43}$ promoter in the genome of BS20, yielding the strain BS20D. Moreover, 2-C-methylerythritol 4-phosphate cytidylyltransferase (IspD) is a phosphocytidyl transferase encoded by ispD and is able to couple 2C-methyl-D-erythritol 4-phosphate (MEP) with cytidine triphosphate (CTP). The relative transcriptional level of $i s p D$ in BS20D was 1.78-times higher than the level of ispD in BS20 (Fig. 2C). The optical density $\left(\mathrm{OD}_{600}\right)$ of BS20D rose to 26 in the first two days, and then quickly dropped to 6 on the fifth day (Fig. 2A). At the same time, the increase in $\mathrm{OD}_{600}$ value also corresponded to an increase in glucose consumption (Fig. 2B). It was shown that the fermentation titer of MK-7 by BS20D was augmented to $353.2 \pm 1.2$ $\mathrm{mg} / \mathrm{l}$, a $10 \%$ increase compared with the production $320.3 \pm 2.5 \mathrm{mg} / \mathrm{l}$ by BS20 (Fig. $2 \mathrm{D}$ ). Then we used $\mathrm{P}_{43}$ promoter to overexpress $i s p F$ in the genome of BS20D, yielding strain BS20DF. In addition, 2-C-methylerythritol 2,4cyclodiphosphate synthase (IspF) is a cyclophosphate synthase encoded by ispF. Although the relative transcriptional level of ispF in BS20DF was 1.3 times higher than that of $i s p F$ in BS20D (Fig. 2C), the fermentation titer of MK-7 by BS20DF was $332.6 \pm 3 \mathrm{mg} / \mathrm{l}$. The result showed a $3.9 \% \mathrm{MK}-7$ titer increase compared with BS20 but a $5.8 \%$ decrease over BS20D (Fig. 2D). This was in contrast to previous research that isp $F$ was more important in the flux control of MEP pathway than ispD [22]. In their study, Li focused on the systematic analysis of consecutive enzymes in the MEP pathway in $B$. subtilis and successfully increased the production of carotenoids. The yield of carotenoids, which also requires IPP as precursor, showed an 11.38-fold increase after additional insertion of gene ispF in ispD-overexpressed strain. The reason for the different influence degree of IspF might be that the key points of metabolic flux are usually different because of the difference in different engineered strains [22].

The strain BS20DFE was obtained by overexpressing ispE with $\mathrm{P}_{43}$ promoter in BS20DF. Although the growth curve of BS20DFE had the same trend as the other engineered strains (Fig. 2A), the highest $\mathrm{OD}_{600}$ value of BSDFE was only 16, and it consumed merely $12 \mathrm{~g}$ of glucose in 6 days (Fig. 2B). MEP was speculated to form IspF-MEP complex with IspF in E. coli although it might lead to an imbalance in the MEP pathway [23]. If the ispD, ispE, and 
A

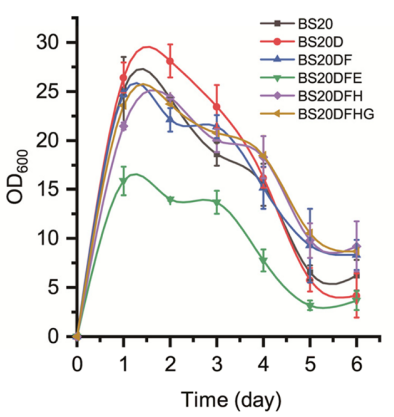

B

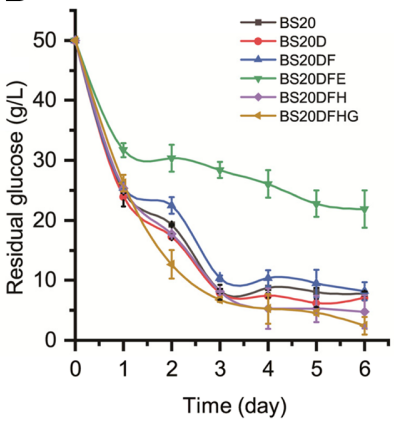

C

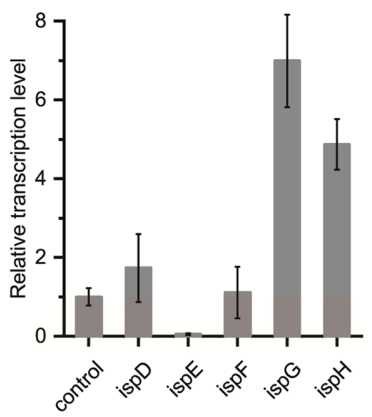

D

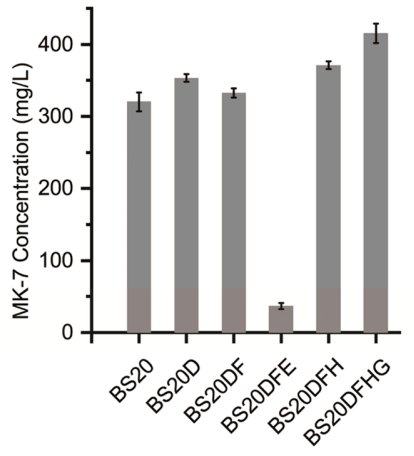

Fig. 2. Trends of cell growth, glucose concentration, relative transcription level and MK-7 production in shake flasks. (A) Growth curves of engineered strains such as BS20, BS20D, BS20DF, BS20DFE, BS20DFH, and BS20DFHG. (B) Glucose concentration of engineered strains during shaking-culture in 250-ml flasks. (C) The relative transcriptional levels of isp-genes (ispD, ispF, ispH, and ispG) which were overexpressed and the relative transcriptional levels of ispE in BS20DFHG, the control gene was $h b s U$ from BS20 genome. The transcriptional level of the isp-genes in BS20DFHG was the same as it was in the individually enhanced strain, just as the transcriptional level of ispD in BS20DFHG was the same as it was in BS20D. (Data shown in Fig. S3) (D)The titers of MK-7 synthesized by strains BS20, BS20D, BS20DF, BS20DFE, BS20DFH, and BS20DFHG in 6 days.

ispF genes are overexpressed in BS20, the intracellular concentration of IspF may increase, and this might lead to the formation of IspF-MEP complex, resulting in an imbalance in the MEP pathway.

IspG is the term for 4-hydroxy-3-methylbut-2-en-1-yl diphosphate (HMBPP) synthase that is encoded by ispG. The overexpression of $i s p G$ has been reported to promote the production of natural products like lycopene and isoprene that utilize IPP as a biosynthetic precursor $[15,19]$. Meanwhile, it was found that activation of the downstream enzyme 4-hydroxy-3-methylbut-2-enyl diphosphate reductase (IspH) could solve the problem of HMBPP accumulation and eliminate the negative effects of isp $G$ overexpression [24]. Thus, we decided to overexpress ispH before isp $G$ to avoid the potential risk of IspG toxicity.

Gene isp $H$ was overexpressed by changing the autologous promoter to $\mathrm{P}_{43}$ promoter in the strain BS20DF, and the obtained strain was named as BS20DFH. The relative transcriptional level of $i s p H$ was 4.87-times higher than that of ispH in BS20 (Fig. 2C). The overexpression of ispH increased MK-7 titer to $370.8 \pm 5.2 \mathrm{mg} / \mathrm{l}$ (Fig. 2D), showing a $15.8 \%$ increase compared with that of BS20. The growth curve of BS20DFH was similar to that of BS20, while the amount and rate of glucose consumption increased (Fig. 2B). Finally, we replaced the promoter of $i s p G$ with $\mathrm{P}_{43}$ promoter in the strain BS20DFH, yielding strain BS20DFHG. Although the strain BS20DFHG did not grow as well as BS20 in the first two days, the $\mathrm{OD}_{600}$ of BS20DFHG in the last four days declined slower than BS20 and eventually stayed at 8.8 (Fig. 2A). Thus, we obtained the highest production of MK-7 as $415 \pm 3.2 \mathrm{mg} / \mathrm{l}$ (Fig. 2D), a $29 \%$ increase compared with BS20 and the relative transcriptional level of isp $G$ in BS20DFHG was 6.9times that in BS20. In a previous work, the $d x s, d x r$, idi, and menA genes were overexpressed in BS168 to improve the production of MK-7 [5]. In this study, we overexpressed the isp-genes of MEP pathway in the BS20 strain to increase the production of MK-7. We supposed that overexpression of isp-genes in MEP pathway had a positive effect on the supply of IPP and accumulation of MK-7.

\section{Optimizing Process Conditions with 300-ml Parallel Bioreactors}

To increase the concentration of MK-7 in bioreactors, we designed a set of orthogonal experiments through the software Minitab to optimize the parameters of the fermentation process, including temperature, aeration and initial $\mathrm{pH}$. The orthogonal experimental design is shown in Table 2 . The experimental design of the three groups of "1", " 2 ", and " 3 " was selected for each factor. The final fermentation titer of the strain BS20DFHG cultured in $300-\mathrm{ml}$ bioreactors was used as the evaluation index. The experimental design and results are shown in Table 3. From the analysis of the extreme difference, the impact intensity of three factors on the fermentation production of $\mathrm{MK}-7$ was $\mathrm{C}$ (initial $\mathrm{pH})>\mathrm{A}$ (temperature) $>\mathrm{B}$ (aeration).

The initial $\mathrm{pH}$ was the most important factor among these three factors. The mean of MK-7 production at $\mathrm{pH}$ 7.0 was $129.55 \mathrm{mg} / \mathrm{l}$, while the mean of the MK-7 output at $\mathrm{pH} 5.5$ was only $59.20 \mathrm{mg} / \mathrm{l}$. It might be related to the fact that the strain BS20DFHG did not grow well when the initial pH was set at 5.5 (Fig. 3). When the initial pH was set at 8.5 , the growth of the strain showed a delay of $10 \mathrm{~h}$ compared with the strains cultured at $\mathrm{pH} 7.0$ (Fig. 3). The trend of growth curves in all conditions was similar to that in shake flasks. When the fermentation temperature was $41^{\circ} \mathrm{C}$, the production of MK-7 was higher than that at the other temperatures, regardless of the other two

Table 2. Experimental factors and levels of fermentation.

\begin{tabular}{cccc}
\hline Level & A-temperature $\left({ }^{\circ} \mathrm{C}\right)$ & B-aeration $(v v m)$ & C-initial $\mathrm{pH}$ \\
\hline 1 & 33 & 0.6 & 5.5 \\
2 & 37 & 1.4 & 7.0 \\
3 & 41 & 2.0 & 8.5
\end{tabular}


Table 3. Effect of three factors on the titer of MK-7.

\begin{tabular}{ccccc}
\hline Run & A & B & C & MK-7 production(mg/l) \\
\hline 1 & 1 & 1 & 1 & $53.54 \pm 2.4$ \\
2 & 1 & 2 & 2 & $90.37 \pm 5.3$ \\
3 & 1 & 3 & 3 & $88.73 \pm 6.1$ \\
4 & 2 & 1 & 2 & $112.72 \pm 5.4$ \\
5 & 2 & 2 & 3 & $136.92 \pm 4.9$ \\
6 & 2 & 3 & 1 & $58.75 \pm 3.3$ \\
7 & 3 & 1 & 3 & $158.66 \pm 6.9$ \\
8 & 3 & 2 & 1 & $185.32 \pm 4.1$ \\
9 & 3 & 3 & 2 & \\
Mean1 & 77.54667 & 108.3067 & 59.3 & \\
Mean2 & 102.7967 & 97.53667 & 129.5533 & \\
Mean3 & 112.79 & 111.0167 & 128.1033 & \\
Range & 35.24333 & 13.48 & 70.35 &
\end{tabular}

factors (Table 3). The aeration, which was expected to play an essential role in fermentation [11], however, didn't have much effect on the biosynthesis of MK-7. As a result, the optimal fermentation scheme could be determined as $41^{\circ} \mathrm{C}, 2 \mathrm{vvm}$ and an initial $\mathrm{pH}$ of 7.0 (A3B3C2). Under these conditions, the engineered strain BS20DFHG produced the highest MK-7 titer of $185.57 \pm 6.3 \mathrm{mg} / \mathrm{l}$ in a 300 - $\mathrm{ml}$ bioreactor.

\section{Constructing a Cell Growth Kinetic Model of BS20DFHG in 50-1 Bioreactor}

After optimizing the three parameters, we determined the optimal fermentation scheme for MK-7 production. According to the optimization experiments of fermentation parameters such as $\mathrm{pH}$ and temperature, the biomass
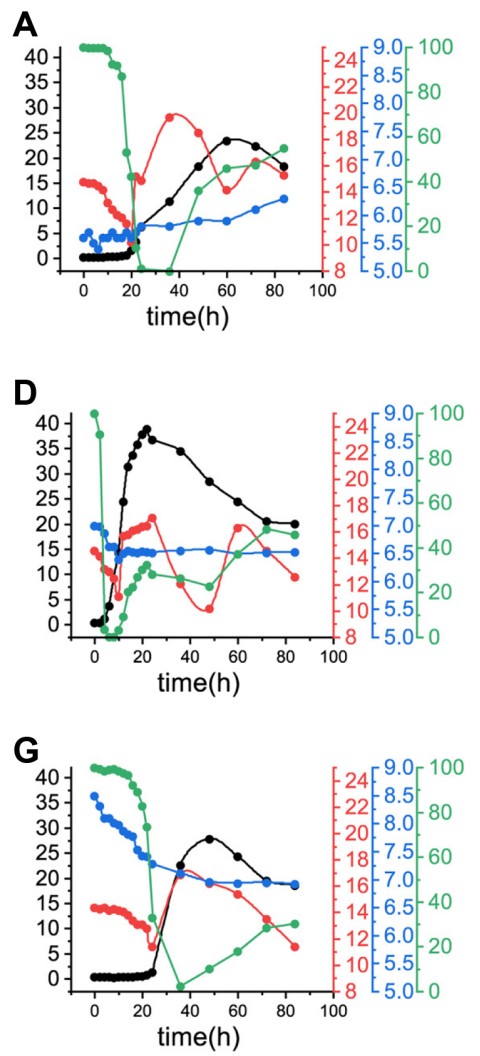

$$
\longrightarrow \mathrm{OD}_{600} \quad \longrightarrow \text { Glucose }(\mathrm{g} / \mathrm{L})
$$
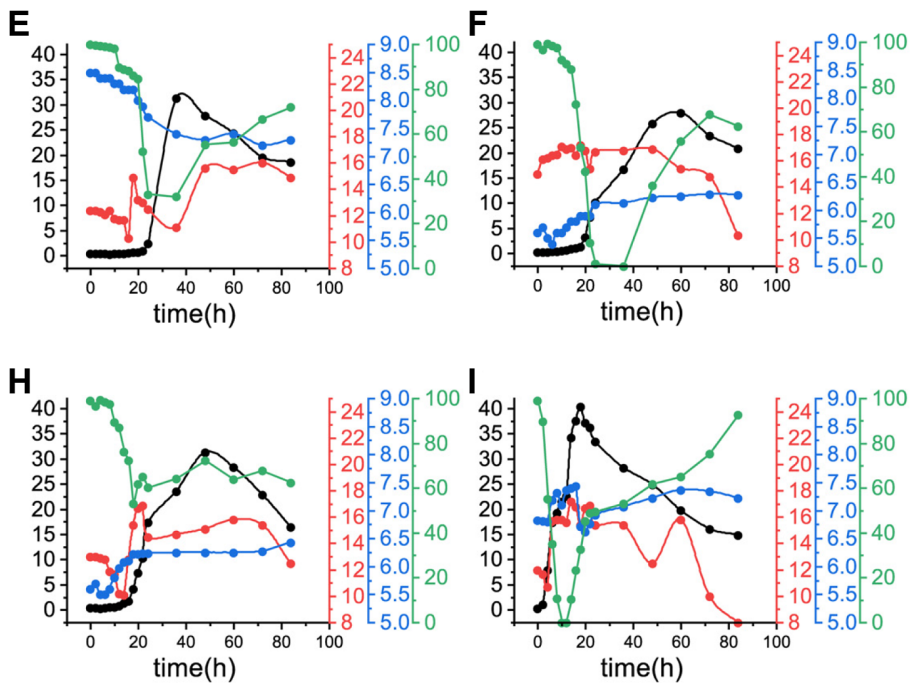

Fig. 3. Trends of cell growth, glucose concentration, $\mathrm{pH}$ and $\mathrm{DO}$ during fed-culture in $300-\mathrm{ml}$ bioreactors. The symbols 'A-I' correspond to Run 1-9 in Table 3. (A) A1B1C1, (B) A1B2C2, (C) A1B3C3, (D) A2B1C2, (E) A2B2C3, (F) $\mathrm{A} 2 \mathrm{~B} 3 \mathrm{C} 1,(\mathbf{G}) \mathrm{A} 3 \mathrm{~B} 1 \mathrm{C} 3,(\mathrm{H}) \mathrm{A} 3 \mathrm{~B} 2 \mathrm{C} 1$, and (I) A3B3C2. 'A1, A2, A3' represented three gradients of culture temperature ranging from 37 to $41^{\circ} \mathrm{C}$ ' $\mathrm{B} 1, \mathrm{~B} 2, \mathrm{~B} 3$ ' represented three gradients of aeration ranging from 0.6 to $2.0 \mathrm{vvm}$. ' $\mathrm{C} 1, \mathrm{C} 2, \mathrm{C} 3$ ' represented three gradients of initial $\mathrm{pH}$ ranging from 5.5 to 8.5 . 


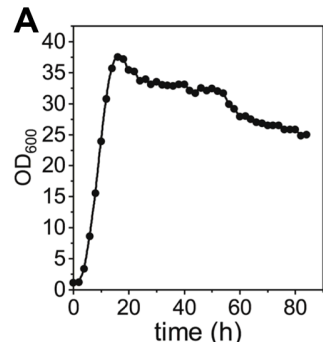

D
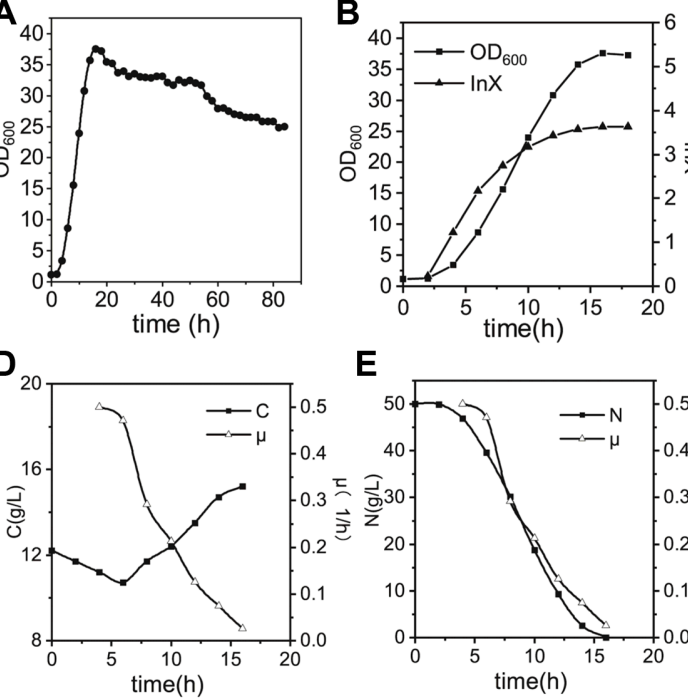

E

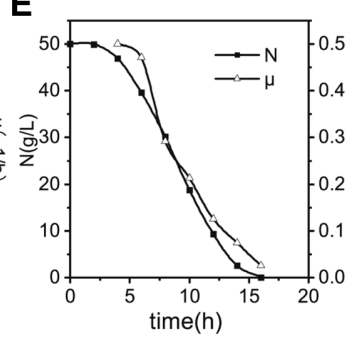

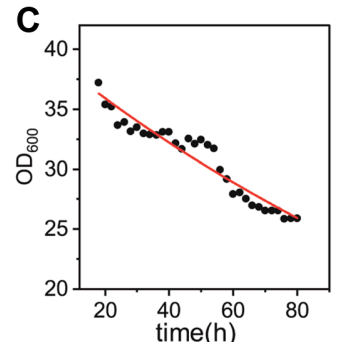

$\mathbf{F}$

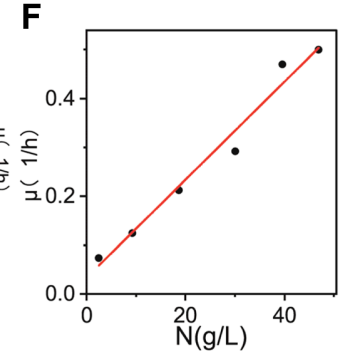

G

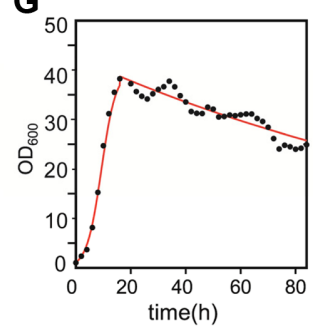

Fig. 4. Diagrams of cell growth kinetic model parameters. The ' $\mu$ ' meant the biomass specific growth rate of the strain BS20DFHG, the ' $C$ ' meant the concentration of carbon source in the bioreactor and the ' $N$ ' meant the concentration of nitrogen source in the bioreactor. The 'InX' meant logarithm of the biomass ' $X$ ' with the constant ' $e$ ' as the base. (A) Growth curve of the engineered strain BS20DFHG in a 50-1 bioreactor. (B) The dots presented the growth curves of the engineered strain BS20DFHG in the first $18 \mathrm{~h}$. The triangles meant the values of ' $\operatorname{lnX}$ '. (C) The dots showed the value of $\mathrm{OD}_{600}$ from $18 \mathrm{~h}$ to $84 \mathrm{~h}$. The red curve showed the fitted curve of $\mathrm{OD}_{600}$ and time. (D) The curve with dots showed the change of the ' $\mathrm{C}$ ' from $0 \mathrm{~h}$ to $16 \mathrm{~h}$. The curve with hollow triangle showed the change of the ' $\mu$ ' from $0 \mathrm{~h}$ to $16 \mathrm{~h}$. (E) The curve with dots showed the change of the ' $\mathrm{N}$ ' from $0 \mathrm{~h}$ to $16 \mathrm{~h}$. The curve with hollow triangle showed the change of ' $\mu$ ' from $0 \mathrm{~h}$ to $16 \mathrm{~h}$. (F) The dots showed the value of the ' $\mu$ ' to different ' $N$ ' and the red line was the fitted line of ' $\mu$ ' and ' $N$ '. (G) The red line showed the simulation curve of cell growth kinetic model and the black solid dots showed values of the experimental $\mathrm{OD}_{600}$ values.

of BS20DFHG at the end of logarithmic growth would affect the final production of MK-7. So, we decided to employ the optimized scheme in 50-1 bioreactor fermentation (Fig. S2). Subsequently, a cell growth kinetic model was constructed to describe and predict the cell growth of BS20DFHG. We separated the growth curve into two parts according to the increase or decrease trend of $\mathrm{OD}_{600}$. As shown in Fig. 4A, the strain BS20DFHG grew rapidly in the first $18 \mathrm{~h}$, and the $\mathrm{OD}_{600}$ slowly decreased in the following 3 days. The growth of the strain BS20DHG could be modeled by an equation in the form of Eq. (1), including the microbial biomass ' $X$ ', the death coefficient ' $K_{d}$ ' and the specific growth rate ' $\mu$ '. In the first $18 \mathrm{~h}$ of the fermentation process (Fig. $4 \mathrm{~B}$ ), the growth curve indicated that there is a relationship between the specific growth rate ' $\mu$ ' and the concentration of limiting nutrients or inhibiting substrates. The negative correlation between ' $\mu$ ' and the concentration of carbon source (C) is presented in Fig. 4D. However, the concentration of glucose $(10 \mathrm{~g} / \mathrm{l})$ is not considered to inhibit bacterial growth based on previous experimental results (Fig. 2A). Therefore, we turned our attention to the consumption of the nitrogen source which could be seen in Fig. 4E, because the nitrogen source usually affects the synthesis of proteins in cells and further affects the growth of strains. As can be seen in Fig. 4F, the concentration of nitrogen source $(\mathrm{N})$ and the ' $\mu$ ' exhibit positive correlation after linear fitting (Eq. (2) $(\mathrm{A}=0.01, \mathrm{~B}=0.0333)$. The dynamic relationship of the ' $\mathrm{N}$ ' and ' $\mathrm{X}$ ' was given in the form of Eq. (3) $\left(\mathrm{Y}_{\mathrm{X} / \mathrm{N}}=0.7276\right)$.

Due to the rich resources in the medium and the exponential growth of the strain BS20DFHG, we assumed the ' $\mathrm{K}_{\mathrm{d}}$ ' $=0$ during the first $18 \mathrm{~h}$, and the growth of BS20DFHG in the 50-1 bioreactor was modeled in the form of Eqs. (1), (2), and (3). In terms of the decline part, Eq. (4) was obtained after integrating Eq. (1) with $\mu=0$ in the following 3 days. It could demonstrate the decline in the growth of BS20DFHG over time. ' $\mathrm{X}_{0}$ ' which was obtained as 40.05 means the initial ' $\mathrm{X}$ ' of the second part and the ' $\mathrm{K}_{\mathrm{d}}$ ' was recognized as 0.0054 (Fig. $4 \mathrm{C}$ ). Thus, the dynamic relationship between the ' $\mu$ ', ' $N$ ' and ' $t$ ' could be described by Eq. (4). So far, we have constructed a cell growth kinetic model of BS20DFHG in a 50-1 bioreactor.

$$
\begin{aligned}
& \frac{\mathrm{dX}}{\mathrm{dt}}=\mathrm{X} *\left(\mu-\mathrm{K}_{\mathrm{d}}\right) \\
& \mu=\mathrm{A} * \mathrm{~N}+\mathrm{B} \\
& -\frac{\mathrm{dN}}{\mathrm{dt}}=\frac{\mathrm{dX}}{\mathrm{dt}} * \frac{1}{\mathrm{Y}_{\mathrm{XNN}}} \\
& X=\mathrm{X}_{0} * \exp \left(-\mathrm{K}_{\mathrm{d}} * \mathrm{t}\right)
\end{aligned}
$$


Next, we carried out fermentation experiments in 50-1 bioreactor to verify the accuracy of the model and observed the highest titer of MK-7 as $242 \pm 5.5 \mathrm{mg} / \mathrm{l}$. As can be seen from Fig. 4G, the fitted curve was close to the experimental values, indicating that the cell growth kinetic model can describe the actual growth process reasonably well. It was indicated that the concentration of nitrogen was significant for the early growth of the strain BS20DFHG. Increasing the initial concentration of soy peptone, a mixed nitrogen source or fed-addition of nitrogen, can be important strategies to further improve the production of MK-7. The cell growth kinetic model of BS20DFHG cannot explain the MK-7 production directly, but it is a powerful tool to explore the relationship between them. From this kinetic model, more thorough experiments can be designed to explore the relationship between MK-7 production and $\mathrm{OD}_{600}$.

In conclusion, after increasing the relative transcription level of isp-genes to increase the flux of the MEP pathway, we obtained the highest production of MK-7 as $415 \pm 3.2 \mathrm{mg} / \mathrm{lin}$ BS20DFHG, a 29\% increase compared with BS20. Then we attempted to figure out the crucial factors of the liquid fermentation process and optimized them by parallel bioreactors. Finally, a cell growth kinetic model was developed to demonstrate the growth of BS20DFHG in a 50-1 bioreactor accurately. It helps us better understand the growth of BS20DFHG in a bioreactor and guide the next step of optimization, and therefore, the established fermentation process paves the way for the industrial production of MK-7 in the future.

\section{Acknowledgments}

This work was financially supported by the National Natural Science Foundation of China (31871784, 31870069, 21676119 and 31671845). Postgraduate Research \& Practice Innovation Program of Jiangsu Province (KYCX18_1786).

\section{Conflict of Interest}

The authors have no financial conflicts of interest to declare.

\section{References}

1. Walther B, Karl JP, Booth SL, Boyaval P. 2013. Menaquinones, bacteria, and the food supply: the relevance of dairy and fermented food products to vitamin K requirements. Adv. Nutr. 4: 463-473.

2. Mahdinia E, Demirci A, Berenjian A. 2017. Optimization of Bacillus subtilis natto growth parameters in glycerol-based medium for vitamin K (menaquinone-7) production in biofilm reactors. Bioprocess Biosyst. Eng. 41: 195-204.

3. Scheiber D, Veulemans V, Horn P, Chatrou ML, Potthoff SA, Kelm M, et al. 2015. High-dose menaquinone-7 supplementation reduces cardiovascular calcification in a murine model of extraosseous calcification. Nutrients 7: 6991-7011.

4. El Asmar MS, Naoum JJ, Arbid EJ. 2014. Vitamin k dependent proteins and the role of vitamin K2 in the modulation of vascular calcification: a review. Oman. Med. J. 29: 172-177.

5. Ma Y, McClure DD, Somerville MV, Proschogo NW, Dehghani F, Kavanagh JM, et al. 2019. Metabolic engineering of the MEP pathway in Bacillus subtilis for increased biosynthesis of menaquinone-7. ACS Synth. Biol. 8: 1620-1630.

6. Baj A, Wałejko P, Kutner A, Kaczmarek Ł, Morzycki JW, Witkowski S. 2016. Convergent synthesis of menaquinone-7 (MK-7). Org. Process Res. Dev. 20: 1026-1033.

7. Mahanama R, Berenjian A, Valtchev P, Talbot A, Biffin R, Regtop H, et al. 2011. Enhanced production of menaquinone 7 via solid substrate fermentation from Bacillus subtilis. Int. J. Food Eng. 7:1-23.

8. Berenjian A, Mahanama R, Talbot A, Biffin R, Regtop H, Valtchev P, et al. 2011. Efficient media for high menaquinone-7 production: response surface methodology approach. N Biotechnol. 28: 665-672.

9. Goodman SR, Marrs BL, Narconis RJ, Olson RE. 1976. Isolation and description of a menaquinone mutant from Bacillus licheniformis. J. Bacteriol. 125: 282-291.

10. Yoshiki T, Hisataka T. 1989. Extracellular production of menaquinone-4 by a mutant of Flavobacterium sp. 238-7 with a detergentsupplemented culture. J. Ferment. Bioeng. 67: 102-106.

11. Berenjian A, Mahanama R, Talbot A, Regtop H, Kavanagh J, Dehghani F. 2014. Designing of an intensification process for biosynthesis and recovery of menaquinone-7. Appl. Biochem. Biotechnol. 172: 1347-1357.

12. Mahdinia E, Demirci A, Berenjian A. 2018. Effects of medium components in a glycerol-based medium on vitamin $\mathrm{K}$ (menaquinone-7) production by Bacillus subtilis natto in biofilm reactors. Bioprocess Biosyst. Eng. 42: 223-232.

13. Cui S, Xia H., Chen T, Gu Y, Lv X, Liu Y, et al. 2020. Cell membrane and electron transfer engineering for improved synthesis of menaquinone-7 in Bacillus subtilis. iScience 23(3): 100918.

14. Cui S, Lv X, Wu Y, Li J, Du G, Ledesma-Amaro R, et al. 2019. Engineering a Bifunctional Phr60-Rap60-Spo0A Quorum-Sensing molecular switch for dynamic fine-tuning of menaquinone-7 synthesis in Bacillus subtilis. ACS Synth. Biol. 8: $1826-1837$.

15. Heider SA, Wolf N, Hofemeier A, Peters-Wendisch P, Wendisch VF. 2014. Optimization of the IPP precursor supply for the production of lycopene, decaprenoxanthin and astaxanthin by Corynebacterium glutamicum. Front Bioeng. Biotechnol. 2: 28.

16. Xue D, Abdallah II, de Haan, IEM, Sibbald, MJJB, Quax WJ. 2015. Enhanced C30 carotenoid production in Bacillus subtilis by systematic overexpression of MEP pathway genes. Appl. Microbiol. Biotechnol. 99: 5907-5915

17. Chou HH, Keasling JD. 2013. Programming adaptive control to evolve increased metabolite production. Nat. Commun. 4: 2595.

18. Abdallah, II, Pramastya H, van Merkerk R, Sukrasno, Quax WJ. 2019. Metabolic engineering of Bacillus subtilis toward taxadiene biosynthesis as the first committed step for taxol production. Front Microbiol. 10: 218.

19. Ye L, Lv X, Yu H. 2016. Engineering microbes for isoprene production. Metab. Eng. 38: 125-138.

20. Yan X, Yu HJ, Hong Q, Li SP. 2008. Cre/lox system and PCR-based genome engineering in Bacillus subtilis. Appl. Environ. Microbiol. 74: 5556-5562.

21. Cabrera-Valladares N, Martinez LM, Flores N, Hernandez-Chavez G, Martinez A, Bolivar F, et al. 2012. Physiologic consequences of glucose transport and phosphoenolpyruvate node modifications in Bacillus subtilis 168. J. Mol. Microbiol. Biotechnol. 22: 177-197.

22. Li M, Hou F, Wu T, Jiang X, Li F, Liu H, et al. 2019. Recent advances of metabolic engineering strategies in natural isoprenoid production using cell factories. Nat. Prod. Rep. 37: 80-99.

23. BitokJK, Meyers CF. 2012.2C-Methyl-d-erythritol 4-phosphate enhances and sustains cyclodiphosphate synthase IspF activity. ACS Chem. Biol. 7: 1702-1710.

24. Li Q, Fan F, Gao X, Yang C, Bi C, Tang J, et al. 2017. Balanced activation of IspG and IspH to eliminate MEP intermediate accumulation and improve isoprenoids production in Escherichia coli. Metab. Eng. 44: 13-21. 